\title{
Comparaison entre turbine Kaplan et groupe bulbe
}

\author{
Comparison between Kaplan turbine and bulb turbine
}

\author{
C. Thirriot
}

\author{
Institut national polytechnique de Toulouse
}

\begin{abstract}
Le développement de l'emploi des turbines bulbes dans l'aménagement des chutes, auparavant équipées en turbines Kaplan, provient de l'amélioration de l'écoulement hydraulique sans changement de direction (augmentation du rendement) et de la réduction des dimensions (réduction du coût de l'investissement) tout en maintenant souplesse de réglage et sécurité.
\end{abstract}

The development of the use of bulb turbines in law head hydraulic plants, previously equipped with Kaplan turbines, has been brought about by an improvement in hydraulic flow without any change of streamline direction (yield increased) and in a reduction of dimensions (reduced investment cost) whilst maintaining safety and adjustment flexibility.

\section{Introduction}

A la différence des technologies modernes électroniques, nucléaires ou aérospatiales qui paraissent ésotériques à Monsieur Tout le Monde, l'industrie des machines hydrauliques paraît faire partie du fond commun de l'humanité, sans barrières de frontières ou de générations. La roue du moulin est familière dès l'enfance à tous les peuples, et l'on trouve tout naturel qu'elle se transforme en turbine Pelton. La machine d'Euler paraît à peine plus surprenante car l'effet de la réaction entraînait déjà les meules à axe vertical des moulins à eaux du Bazacle sur la Garonne à Toulouse, au XVII ${ }^{e}$ siècle.

L'art de l'hydraulique est le plus vieux métier du monde et les prouesses d'aujourd'hui, dans les gains de rendement, sont étayées par des siècles d'expériences et de sagesse mûries autant par les échecs que par les intuitions des inventeurs.

Comme dans beaucoup d'autres pays, en France le mariage de l'eau et de l'électricité à la fin du siècle dernier, a été décisif pour stimuler les progrès des métiers de fabricants de turbines et de pompes.

Peu à peu, des essais de formes hétéroclites de roues, a émergé le classement unificateur des machines à réaction, qu'elles soient motrices (turbines) ou génératrices d'énergie pour le fluide (pompes) grâce au concept de vitesse spécifique.

Pour les fortes vitesses spécifiques, sont en compétition les roues Kaplan et les systèmes bulbes.

La figure I rappelle que la distinction essentielle entre turbine Kaplan et turbine bulbe réside dans l'orientation de l'arbre de la roue, l'axe est vertical pour la turbine Kaplan, il est horizontal pour le groupe bulbe.

Si l'on se souvient que le brevet de Kaplan consistait à rendre orientable les pales d'une turbine hélice, la turbine bulbe est de la famille Kaplan à axe horizontal.

Certains auteurs parlent, d'ailleurs, dans les comparaisons, de turbine à axe vertical et de turbine à axe horizontal. Pour notre part, dans la suite, nous conserverons les dénominations familières de Kaplan et bulbe. 


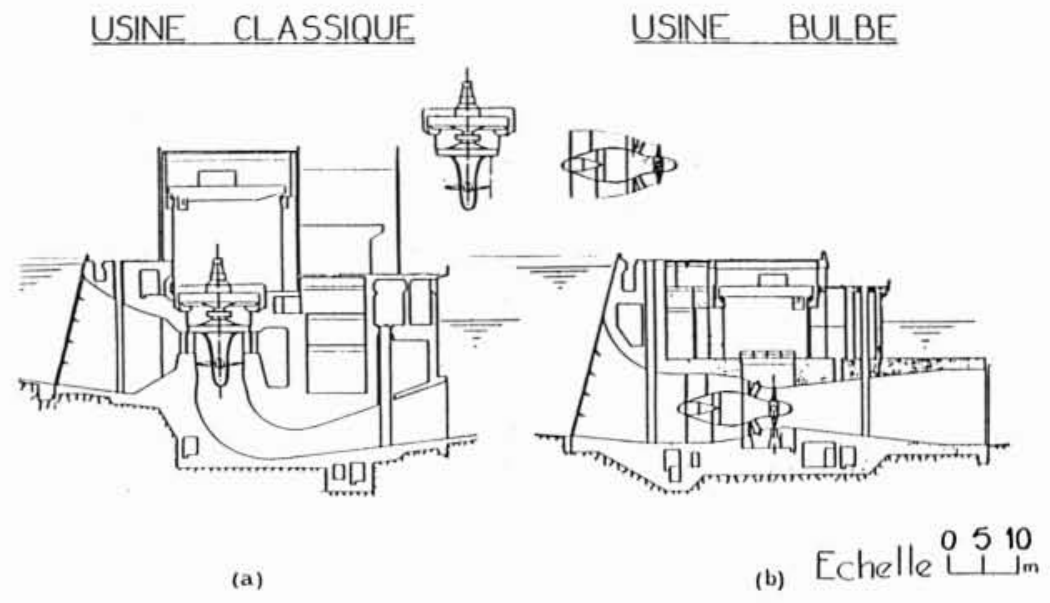

\section{Histoire et statistique}

Comme le relate J. Cotillon, l'évolution vers les groupes à écoulement axial horizontal a été marquée par trois brevets, acquis par Escher Wyss, sur une très courte période.

Le 27 juillet 1930, est déposé le brevet d'une turbine tubulaire, à conduit en S, inventée par G. Khune.

Le 31 août 1933, dépôt du brevet de l'inventeur J. Haefele, de la première turbine à écoulement axial horizontal.

Quatre mois plus tard, le 27 décembre 1933, est déposé le projet d'un premier groupe compact bulbe, inventé par Huguenin.

Le brevet homologué le 15 octobre 1934, est exploité par Arno Fisher, qui, en août 1936, met en service deux groupes de $168 \mathrm{~kW}$ à Rostin qui fonctionneront sans incident jusqu'en janvier 1942 (Cotillon, 1973).

En France, les premières unités de groupes bulbes de puissance appréciable ont été mis en service il y a plus de vingt ans, à Saint-Malo (1960), sur l'usine marémotrice de la Rance (1966) (la première du monde) et à Pierre Bénite (1966) près de Lyon sur le Rhône en profitant des essais à échelles déjà industrielles à Cambeyrac, Argentat et Beaumont-Monteux. A l'époque, les turbines Kaplan avaient déjà acquis leurs lettres de noblesse et atteint un perfectionnement technique peu dépassé depuis. Elles existaient déjà en plusieurs centaines d'exemplaires à travers le monde. Aujourd'hui, les groupes bulbes ont grignoté le marché basse chute fief des turbines Kaplan. Dans une étude statistique récente, T. Strohl dénombre 87 groupes bulbes, contre 48 turbines Kaplan ou assimilées sur 135 sîtes de plus de $5 \mathrm{MW}$ de puissance et de moins de $20 \mathrm{~m}$ de hauteur de chute équipés dans le monde de 1970 à 1984 (figure 2).

Comment expliquer cette suprématie qui s'affirme, des jeunes bulbes contre les honorables Kaplan?
La raison est surtout d'ordre économique : à condition de sites identiques, les groupes bulbes sont moins chers à l'investissement et légèrement de meilleure productivité au fonctionnement. Bien sûr, cette affirmation est à nuancer suivant certaines circonstances locales favorables aux turbines Kaplan.

L'innovation évidente pour le béotien est le caractère compact du groupe bulbe puisque turbine et alternateur forment un tout, la machine électrique se nichant dans le gros bulbe étanche en amont de la roue. L'exigüité relative de ce logement a catalysé les progrès sur l'alternateur dont nous ne parlerons pas ici, nous cantonnant aux aspects hydrauliques et à leur conséquence.

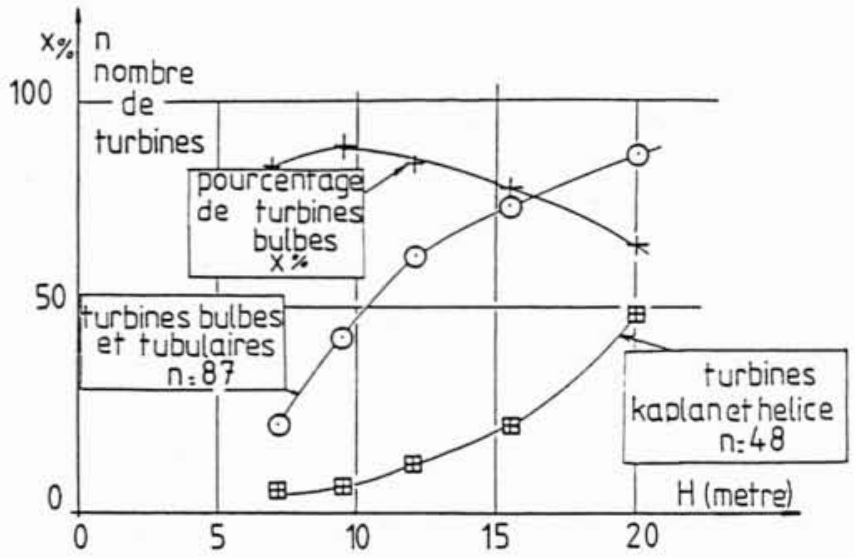

2. Statistiques des turbines commandées entre 1970 et 1984 pour 135 aménagements de plus de $5 \mathrm{MW}$. 


\section{Problèmes d'aménagement des basses chutes}

Pour examiner de la manière la plus objective possible la concurrence entre groupe bulbe et turbine Kaplan, essayons d'abord de définir, de manière simple et concrète, le problème qui constitue le champ de compétition.

Il s'agit d'équiper un sîte à faible chute $H_{v}$ (moins de 20 mètres en général) et à débit $Q$ important (s'il s'agit d'aménagement à puissance de quelques mégawatts).

Mais tout autant que les valeurs nominales $H_{0}$ et $Q_{i}$, vont compter les fluctuations de ces paramètres. A Pierre Bénite par exemple, la chute peut varier de $3,35 \mathrm{~m}$ à 12,40 $\mathrm{m}$ et le débit de $1400 \mathrm{~m}^{3} / \mathrm{s}$ à $150 \mathrm{~m}^{3} / \mathrm{s}$.

Le critère global à prendre en compte, en définitive, sera la production annuelle d'énergie électrique.

Cette production annuelle, $E$, peut être estimée en partant des distributions statistiques concomitantes de débit et de chute, en faisant intervenir le rendement $\eta$ :

$$
E=\int_{0}^{T} \eta \rho g Q H d t
$$

Or, le rendement $\eta$, élément de pondération du produit $Q H$, va dépendre aussi, de manière sensible, des variations de $Q$ et $H$ et donc la variation de $\eta(Q . H)$ va être très importante dans la comparaison entre turbine Kaplan et groupe bulbe, lorsque les plages de variations de $H$ et de $Q$ sont assez étendues. Il ne suffira pas de comparer les rendements maximaux obtenus pour chacune des solutions, au cœur de la colline de rendement, pour les conditions optimales de fonctionnement. Il faudra aussi intégrer les aléas hydrologiques concernant les apports.

Le problème étant ainsi posé, restent à faire les choix des paramètres essentiels de l'aménagement, le type de machines, leur nombre et la dimension caractéristique d'une unité qui sera le diamètre $D$ de la roue.

Pour le type de machine, on admettra que pour les basses chutes considérées, l'affaire est entendue, il ne peut s'agir que de Kaplan ou de bulbes.

Pour le choix du nombre d'unités, les contingences particulières du sîte peuvent intervenir. Mais, en général, on est guidé par la recherche de l'optimum économique compte tenu des possibilités technologiques.

Pour ce qui concerne les Kaplan, les Soviétiques, il y a déjà vingt ans, avaient réalisé des roues jusqu'à $9,30 \mathrm{~m}$. En Chine, à Tchi Li Long, l'aménagement fait état de diamètre de $8,00 \mathrm{~m}$, sous une chute de $14 \mathrm{~m}$. Et plus récemment, des réalisations font intervenir des roues de $11 \mathrm{~m}$. Par exemple, la réalisation chinoise de Gezhouba où, en 1981, furent mis en service deux groupes de $170 \mathrm{MW}$ et de $11,3 \mathrm{~m}$ de diamètre, complétés en 1982 puis 1983 par 5 groupes de $125 \mathrm{MW}$ et de 10,3 m de diamètre.

En France, la Société Neyrpic à Grenoble, construit actuellement avec son licencié MEP, des turbines Kaplan de $8,60 \mathrm{~m}$ de diamètre de roue pour les groupes de la centrale de Porto Primavera (Brésil).

Pour ce qui concerne les bulbes, il y a vingt ans passés, Neyrpic réalisait déjà, pour le compte de l'Electricité de France, des roues de $5,35 \mathrm{~m}$ pour l'usine marémotrice de la Rance et des roues de $6,10 \mathrm{~m}$ pour Pierre-Bénite.
Actuellement, l'optimum de la dimension du diamètre semble se situer entre 6 et $9 \mathrm{~m}$. La société grenobloise a construit 8 roues de $7,40 \mathrm{~m}$, installées à Rock Island aux U.S.A., mais des études de faisabilité ont été exécutées sur une roue de $9,00 \mathrm{~m}$ pour une usine marémotrice.

Il semble que les roues les plus grosses qui tournent actuellement, sont celle de Wall See, en Autriche, qui ont un diamètre de $7,80 \mathrm{~m}$ (légèrement supérieur au diamètre de 7,70 m de l'usine de Racine aux USA).

A partir de ces conditions, on peut envisager le choix économique du nombre d'unités. Nous serons guidés par l'étude fine faite par P. Cazenave (1963) pour l'usine de Pierre-Bénite, de la Compagnie nationale du Rhône.

D'après les spécialistes de Neyrpic, la masse des turbines varie à peu près comme $D^{5 / 2}$. Le prix des turbines (hors études) varie un peu moins vite que la masse, environ comme $D^{2}$, c'est-à-dire comme le débit $Q_{\mu}$ qui traverse la roue. Le nombre de groupes $n=Q^{\prime} Q_{\text {u }}$ varie donc comme $1 / D^{2}$. Le prix total hors étude ne dépend donc pas du nombre de groupes. Le coût de l'étude n'intervenant qu'une fois, il y a donc une tendance favorable au choix d'un nombre élevé de groupes.

Pour ce qui concerne l'alternateur, sa masse unitaire varie en fonction du couple $C$ suivant $C^{2 / 3}$.

$$
\text { Or, } \quad C=P_{u} / 2 \pi N
$$

avec $P_{u}$ : puissance unitaire

$N$ : fréquence de rotation.

La règle de similitude hydraulique impose la constance de

$$
\frac{N Q_{u}^{t / 2}}{H^{3 / 2}}
$$

D'où, à $H$ fixé, $N$ varie comme $Q_{u}^{-1 / 2}$.

Comme $P_{u}=\eta \rho g H Q_{u}$, il découle que $C$ varie comme $Q_{i}^{3 / 2}$ et la masse unitaire d'un alternateur varie donc comme $Q_{\text {. }}$.

Soit $n$ le nombre de groupes. $Q_{\|}=Q / n$.

La masse totale des alternateurs est proportionnelle à $n Q_{u}=Q$.

Donc, la masse des alternateurs est peu sensible au fractionnement, au choix du nombre $n$ de groupes.

Pour le génie civil, on peut se donner une idée grossière du volume en considérant que la longueur de la centrale (suivant l'écoulement) varie comme la dimension caractéristique du groupe, soit le diamètre de la roue $D$, la largeur comme $n D$ et la profondeur à peu près comme $D$. Ainsi, approximativement, le volume de terrassement varie comme $n D^{3}$. Or, $D$ varie comme $1 / \sqrt{n}$. Le volume de terrassement varie donc comme $1 / \sqrt{n}$. En résumé, il semble toujours économique de choisir le plus grand nombre possible de groupes.

Mais P. Cazenave montre que pour optimiser le nombre de groupes, il faut approfondir l'analyse technico-économique et examiner en particulier la part des auxiliaires.

Pour Pierre Bénite, la considération de l'ensemble des facteurs a conduit à choisir la solution de quatre groupes $(n=4)$.

En général, dans la comparaison entre Kaplan et bulbes, on arrive au même nombre de groupes, quel que soit le type de turbine (mais il se peut qu'il existe des circonstances très particulières, par exemple d'encombre- 
ment dans un sîte resserré où les nombres de groupes seraient différents).

Supposons donc qu'on aboutisse au même nombre de groupes. Pour décider du choix du type de turbine, reste donc à comparer avantages et inconvénients intrinsèques des Kaplan et des bulbes.

Cette comparaison peut être faite en deux temps. En effet, nous avons déjà insisté sur le fait que les aménagements basse chute vont devoir fonctionner sous des charges et des débits fortement variables.

Dans une première étape, nous définirons les turbines pour des conditions de charge et de débit constantes, désignées comme conditions nominales $\left(H_{0}, Q_{\mu o}\right)$.

Dans une deuxième étape, nous examinerons le comportement des solutions retenues devant les fluctuations de débit et de charge.

\section{Expérience des constructeurs et similitude}

Revenons à l'étape numéro $1 . H_{o}$ et $Q_{u}$ étant fixés, l'optimisation consiste à obtenir la puissance maximale, c'est-à-dire le rendement maximal pour le moindre coût, c'est-à-dire le diamètre le plus petit.

Là intervient alors ce qui fait toujours l'admiration de l'universitaire que je suis, l'énorme expérience des constructeurs de machines hydrauliques.

Une expérience qui est riche d'innombrables essais sur modèles réduits et de centaines de réalisations sur près d'un siècle, qui se fortifie chaque année par le travail patient au bureau d'étude, l'observation attentive en laboratoire, la critique pragmatique en atelier et la vérification lors des essais de réception.

Ce savoir-faire, ce métier, ces dessins, ces formes qui donnent naissance à une machine hydraulique robuste, économique et performante, a été organisé, généralisé et mémorisé, grâce à la similitude hydrodynamique et la définition de grandeurs spécifiques.

Bien conscients de la vertu des produits sans dimension du théorème de Vaschy-Buckingham, les hommes des turbines et des pompes ont tout de même conservé des dimensions à leurs grandeurs spécifiques.

Cela surprend un peu le mécanicien des fluides universitaire. Cela complique un peu le dialogue entre spécialistes utilisant des systèmes d'unités différents, mais l'énorme avantage est le caractère concret des grandeurs considérées pour la machine de référence. Un petit regret cependant, c'est que la définition de la vitesse spécifique $N_{s}$ varie du langage du turbinier à celui du pompier. Pour l'un, dont la machine, la turbine, fournit de l'énergie mécanique, c'est la puissance $P$ qui intervient parce que caractéristique de l'effet recherché. Alors, la vitesse spécifique a pour expression :

$$
N_{s}=\frac{N P^{1 / 2}}{H^{5 / 4}}
$$

avec $N$ : fréquence de rotation en tours par minute,

$P$ : puissance en $\mathrm{kW}$

$H$ : hauteur de chute en mètre.

Cette vitesse spécifique $N_{s}$ est bien concrète, puisque c'est la vitesse d'une turbine fournissant une puissance de $1 \mathrm{~kW}$ sous une chute de $1 \mathrm{~m}$.

Et chaque constructeur a pour chaque type de turbine, dans ces dossiers, cette turbine fictive aux performances optimales dont le $N_{s}$ optimal va être, par le miracle de la similitude, la clef de réalisation de machines de toutes tailles et parfois gigantesques.

Pour le fabricant de pompe, l'effet recherché est la circulation d'un débit, la puissance mise en œuvre n'étant qu'un moyen. Aussi, la vitesse spécifique s'exprimerat-elle par l'expression :

$$
N_{s}^{\prime}=\frac{N Q^{1 / 2}}{H^{3 / 4}}
$$

avec $Q$ en $\mathrm{m}^{3} / \mathrm{s}$.

Avec les unités habituelles américaines, les vitesses spécifiques sont 51,6 fois plus fortes que celles exprimées dans le système européen.

Si on est gêné par la différence de définition pour les turbines et les pompes, on peut tenter le rapprochement en utilisant dans l'expression de la puissance $P=\eta \rho g H Q$ une valeur moyenne du rendement $\eta$ (par exemple $\eta=$ $0,9)$.

Alors :

$$
N_{s}^{\prime}=\frac{N P^{1 / 2}}{H^{5 / 4}} \frac{1}{(\eta \rho g)^{1 / 2}} \neq \frac{N_{s}}{3}
$$

(en faisant attention à l'évaluation de $P$ en watt).

Ces différences de définitions dues à l'habitude et au pragmatisme des constructions spécialisées, complique l'examen d'une machine hermaphrodite, comme le groupe bulbe qui déjà sur l'usine marémotrice de la Rance se comportait, tantôt comme turbine, tantôt comme pompe.

L'inconvénient disparait si on utilise dans la comparaison une notion de vitesse spécifique vraiment adimensionnelle, directement issue de l'analyse dimensionnelle, telle que :

$$
N^{*}=\frac{N P^{1 / 2}}{\rho^{1 / 2}(g H)^{5 / 4}} \text { ou } N^{* \prime}=\frac{N Q^{1 / 2}}{(g H)^{3 / 4}}
$$

Alors, $N^{*}$ et $N^{* \prime}$ ne diffèrent plus que de l'épaisseur de l'écart de $\eta^{1 / 2}$ par rapport à 1 .

Mais revenons à notre propos, la comparaison entre turbine Kaplan et groupe bulbe.

Fort de son expérience, chaque constructeur a donc un dessin de turbine correspondant au $N_{s}$ optimal qui assure, pour les conditions nominales de fonctionnement, la conjugaison heureuse des inclinaisons des aubes directrices du distributeur et des pales de la roue.

Une enquête statistique montre, comme l'indique la figure 3 empruntée à J. Gautheron et N. Roche (1982), que cette vitesse spécifique optimale dépend néanmoins de la hauteur de chute. En moyenne:

$$
N_{s_{\text {opt }}} \sim H^{-1 / 2}
$$

A partir des résultats statistiques rassemblés par Strohl, nous avons examiné plus en détail l'évolution de $N$ pour les groupes bulbes et les turbines Kaplan dans la zone de basse chute $(H<20 \mathrm{~m})$ et nous avons ainsi pu construire le graphe de la figure 4,qui montre une nette décroissance 


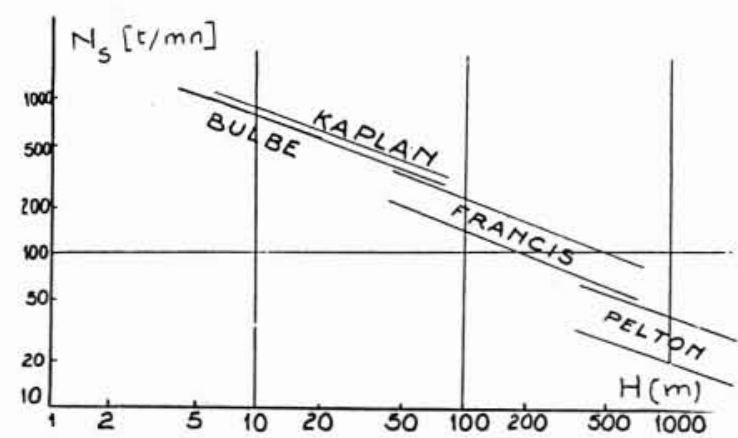

3.

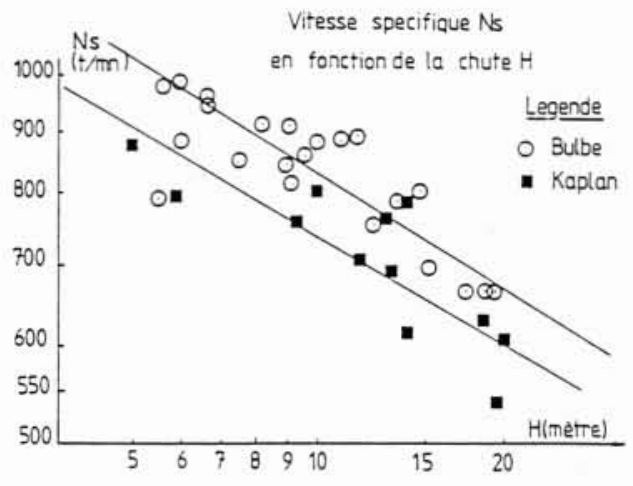

4. Vitesse spécifique en fonction de la chute $H$.

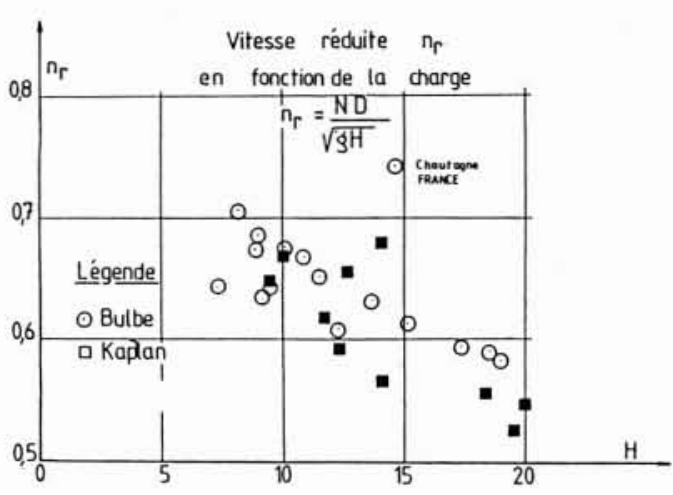

5. Vitesse réduite $\eta$, en fonction de la charge. du $N_{s}$ lorsque $H$ croît de 5 à $20 \mathrm{~m}$. Les résultats afférents aux Kaplan sont nettement en-dessous de ceux concernant les bulbes pour les très faibles chutes et sembleraient s'en rapprocher au voisinage de $H=20$.

Par curiosité, nous avons aussi construit le graphe (figure 5) correspondant aux valeurs réellement adimensionnelles faisant intervenir diamètre de roue et hauteur de chute :

$$
n,=\frac{N D}{\sqrt{g H}}
$$

On obtient alors un meilleur groupement. Les points afférents aux deux types de turbine sont mieux mêlés, mais on note aussi une décroissance de la vitesse relative $n_{r}$ avec l'augmentation de la chute. Ceci n'est pas une remise en question de la similitude, mais traduit le fait qu'il y a de petites modifications de la morphologie des machines lorsque la hauteur de chute augmente.

Il ne faut pas perdre de vue que la vitesse relative $n$, est déterminée après choix du diamètre de la roue, alors que la vitesse spécifique $N_{s}$ est un élément de prédétermination de la machine, et donc, par conséquent, du diamètre. (Mais bien entendu si le choix est l'aboutissement d'un procédé itératif, les deux notions peuvent jouer approximativement le même rôle).
Puisque nous avons introduit le diamètre de la roue, nous pourrions parler d'une notion familière aux turbiniers, le diamètre spécifique $D_{s}$, en utilisant les conditions de similitude, c'est-à-dire les produits sans dimension avec les notations suivantes :

$\begin{array}{cc}\text { Prototype } & \text { Modèle ou machine de référence } \\ N & N_{s} \\ D & D_{s} \\ H & H_{s} \\ P & P_{s}\end{array}$

Considérons les produits sans dimension :

$$
\frac{N D}{\sqrt{g H}} \text { et } \frac{P}{\rho N^{3} D^{5}}
$$

La condition de similitude conduit à :

$$
\frac{D_{s}^{2} H_{s}^{3 / 2}}{P_{s}}=\frac{D^{2} H^{3 / 2}}{P}
$$

D'où l'expression du diamètre spécifique pour la machine fictive spécifique correspondant à une puissance $P_{s}=1 \mathrm{~kW}$ et $H_{s}=1 \mathrm{~m}$ :

$$
D_{s}=D \frac{H^{3 / 4}}{P^{1 / 2}}
$$




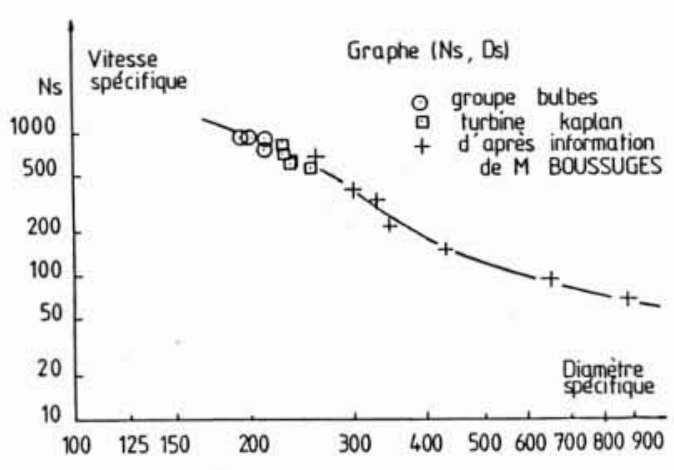

6.

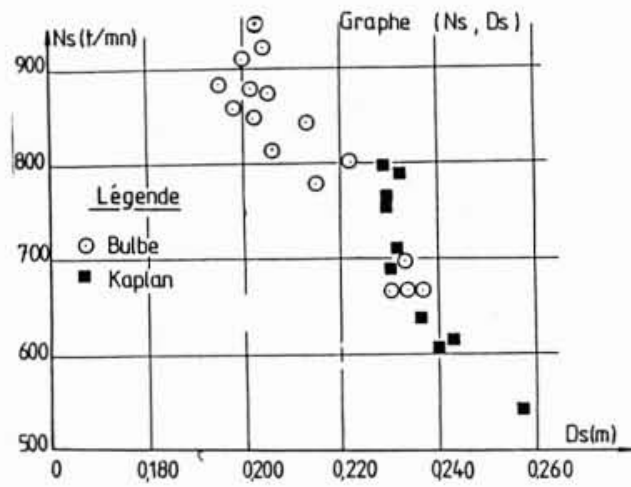

7.

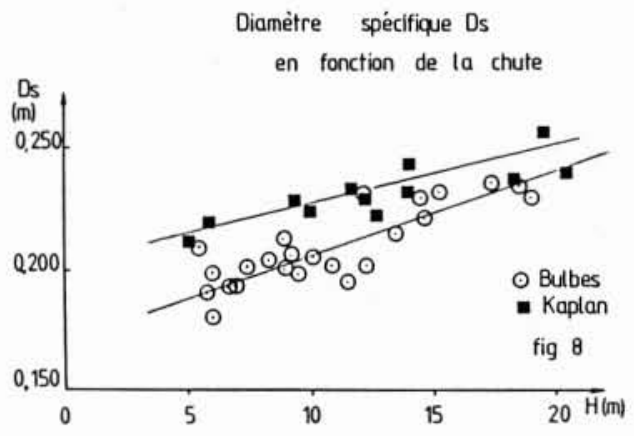

8.

\section{Diamètre spécifique pour turbine Kaplan et groupe bulbe}

Toujours à partir des informations recueillies par Strohl, nous avons calculé les diamètres spécifiques afférents aux machines pour lesquelles nous avons déjà calculé les vitesses spécifiques.

A partir de nos résultats de calcul, nous avons construit deux types de graphes:

a) des graphes $\left(D_{s}, N_{s}\right)$, figures 6 et 7 ,

b) un graphe $\left(D_{s}, H\right)$, figure 8 .

Pour la construction de la figure 6, nous avons utilisé des informations de M. Boussuges.

$\mathrm{Ce}$ graphe synthétique permet de situer turbines Kaplan et bulbes par rapport à l'ensemble des turbines à réaction.

Très grossièrement, la vitesse spécifique $N$, varierait comme le diamètre spécifique au carré, mais le taux de variation $d \log N_{s} / d \log D_{s}$ semble plus faible pour les parties extrêmes du graphe.

Par exemple, si nous examinons plus en détail sur la figure 7 les résultats afférents aux turbines Kaplan et bulbe, nous notons une tendance moyenne commune telle que :

$$
N_{s} \sim D_{s}^{1.8}
$$

Localement, sur la plage de variation de $D_{i}$, on peut d'ailleurs se contenter d'une régression linéaire.

A noter encore que les résultats afférents aux Kaplan considérées seuls font apparaître une très faible variation du diamètre spécifique (de 230 à $240 \mathrm{~mm}$ ) pour une dispersion assez grande de la vitesse spécifique (de 600 à $800 \mathrm{t} / \mathrm{mn}$, si on exclut le point extrême).

Avec la considération de l'évolution du diamètre spécifique en fonction de la chute (figure 8), apparaît une excellente concentration des résultats afférents aux Kaplan qui s'ordonnent autour d'une droite de pente faible, mais significative de l'influence de la hauteur de chute $\left(d D_{s} / d H \simeq 0,025\right)$.

Les valeurs afférentes aux groupes bulbes, un peu plus dispersées, montrent, dans leur tendance moyenne, une sensibilité plus forte à la variation de chute $\left(d D_{2} / D H \simeq\right.$ $0,035)$. La plus grande dispersion résulte essentiellement du fait que les constructeurs n'ont pas développé des tracés représentant les différents $N$, avec autant de finesse que pour les Kaplan et surtout les Francis, pour la raison que le rendement nominal n'est pas du tout le seul critère et que la productivité est moins sensible au $N_{s}$. Les diamètres spécifiques des groupes bulbes se rapprochent de ceux des Kaplan pour $H$ proche de $20 \mathrm{~m}$. Mais pour $H<10$, l'écart sur les diamètres spécifiques est de l'ordre de $12 \%$, ce qui affirme le net avantage des groupes bulbes sur les turbines Kaplan au point de vue encombrement et donc coût.

Déjà, dans son analyse des projets de Pierre-Bénite, P. Cazenave avait insisté sur cette supériorité des groupes bulbes. Il a montré que le choix optimal du diamètre de $6,10 \mathrm{~m}$ pour le groupe bulbe, tournant à $83,3 \mathrm{t} / \mathrm{mn}$, avait été fait après l'étude complète de 6 projets de diamètres différents, allant de $5,70 \mathrm{~m}$ à $6,70 \mathrm{~m}$. La turbine Kaplan équivalente, couplée à un alternateur de même puissance $20000 \mathrm{kVA}$ exigeait un diamètre de $7 \mathrm{~m}$ qui représente une 
augmentation de $13 \%$ de la dimension linéaire, chiffre bien en accord avec notre graphe statistique de la figure 8 .

Le gain de masse pour une unité de Pierre-Bénite, est alors donné par le tableau.

\begin{tabular}{|l|c|c|c|}
\hline & $\begin{array}{c}\text { Kaplan } \\
\text { verticale }\end{array}$ & $\begin{array}{c}\text { Bulbe } \\
\text { horizontal }\end{array}$ & Gain de masse \\
\hline Turbine & $720 \mathrm{t}$ & $575 \mathrm{t}$ & $145 \mathrm{t}(20 \mathrm{z})$ \\
\hline Alternateur & $270 \mathrm{t}$ & $145 \mathrm{t}$ & $125 \mathrm{t}(45 \mathrm{z})$ \\
\hline Groupe complet & $990 \mathrm{t}$ & $720 \mathrm{t}$ & $270 \mathrm{t}(27 \mathrm{z})$ \\
\hline
\end{tabular}

\section{Cavitation}

Si on considère le produit $D N$, d'après les valeurs indiquées par P. Cazenave, il est sensiblement le même pour la turbine Kaplan et le groupe bulbe, ce qui signifie que la vitesse périphérique est la même en bout de pale $(V=\pi N D \simeq 26 \mathrm{~m} / \mathrm{s}$ ). Cette valeur est importante pour décider de l'effet de la cavitation sur le rendement. Une valeur égale de la vitesse périphérique pour les deux types de machines, peut laisser augurer d'éviter le risque de cavitation en bout de pale en maintenant le point de fonctionnement nominal au cœur de la colline de rendement. Mais il ne faut pas oublier que c'est la cavitation de moyeu qui dimensionne souvent les groupes bulbe car c'est elle qui définit la limite d'altération des rendements.

P. Cazenave approfondit l'examen de cette question de cavitation en considérant pour une même cote de fond de fouille, la position des deux roues en compétition dans le projet de Pierre-Bénite. Aux points hauts des roues, la pression statique est de $17 \mathrm{~m}$ pour la turbine Kaplan et de $24 \mathrm{~m}$ pour le groupe bulbe.

Cet avantage du groupe bulbe se retrouvera dans l'ensemble des aménagements de basse chute.

Dans la pratique, les groupes bulbe sont calés aussi juste que les turbines Kaplan et les pales de bulbe aussi bien que de Kaplan sont toujours en acier inoxydable (ou parfois en bronze-aluminium pour les petites dimensions).

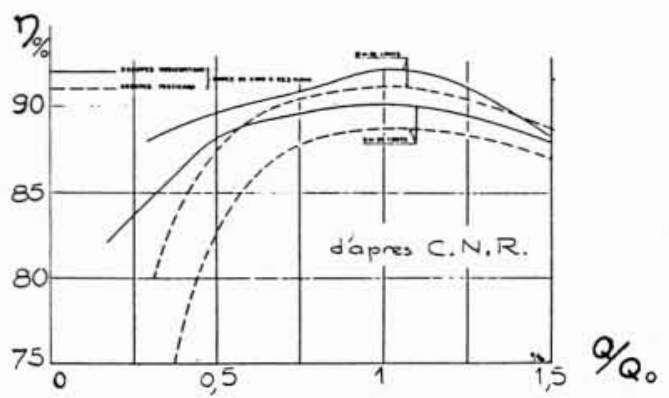

9. Courbes de rendement à chute constante.
Du point de vue du fonctionnement, la roue du groupe bulbe, calée très bas, sera moins sensible aux effets de la cavitation apparaissant à l'emballement, qui provoque une dissymétrie d'effort.

Avec cet aperçu sur la cavitation, nous venons d'aborder les aspects hydrauliques que nous allons maintenant considérer en détail pour juger des rendements.

\section{Ecoulements et pertes de charge}

Le gros avantages hydraulique du groupe bulbe horizontal, c'est l'écoulement quasi rectiligne qui diminue considérablement les pertes d'énergie par rapport à la turbine Kaplan verticale où il $\mathrm{y}$ a changement de direction de l'écoulement à l'amont à la sortie de la volute et à la sortie dans l'aspirateur qui restitue l'eau à l'aval.

Pour juger de l'effet spécifique de la disposition verticale ou horizontale, Martin et Giraud (1960) ont réalisé des essais avec une même roue pour un débit spécifique $Q / D^{2} \sqrt{H}=2,1 \mathrm{~m}^{3} / \mathrm{s}$. La distribution des pertes d'énergie était la suivante :

\begin{tabular}{|l|c|c|c|c|}
\hline & Entrée & Roue & Aspirateur & $\begin{array}{c}\text { Rendesent } \\
\text { global : }\end{array}$ \\
\hline Axe horizontal & 1,7 & 4,2 & 5 & 89 \\
\hline Axe vertical & 5,4 & 1,0 & 9 & 84,4 \\
\hline
\end{tabular}

Il faut bien sûr remarquer, que la mauvaise performance dans la roue elle-même, en position à axe horizontal, tenait au fait que les expérimentateurs avaient utilisé une roue qui n'avait pas le dessin spécifique d'une roue bulbe.

Des expériences systématiques ont permis d'apprécier la perte de charge dans l'aspirateur en fonction de l'énergie cinétique à la sortie de l'aspirateur :

$$
\xi_{a}=\alpha \frac{V^{2}}{2 g}
$$

$\alpha=1,4$ pour les groupes bulbe,

$\alpha=3$ pour les turbines Kaplan avec un aspirateur coudé (Lathuile).

\section{Rendement et production annuelle d'énergie}

Pour donner une idée des valeurs absolues des rendements, nous reviendrons encore une fois à l'exemple de Pierre-Bénite. La figure 9 montre que, d'après les essais sur modèle, sous la hauteur de chute nominale, le groupe bulbe, à près de $92 \%$ de rendement, gagne $1 \%$ environ sur la turbine Kaplan et l'avantage s'accentue pour une chute plus faible. A $8 \mathrm{~m}$, l'écart est de $1,5 \%$ au bénéfice du groupe bulbe. La même figure montre que le rendement 


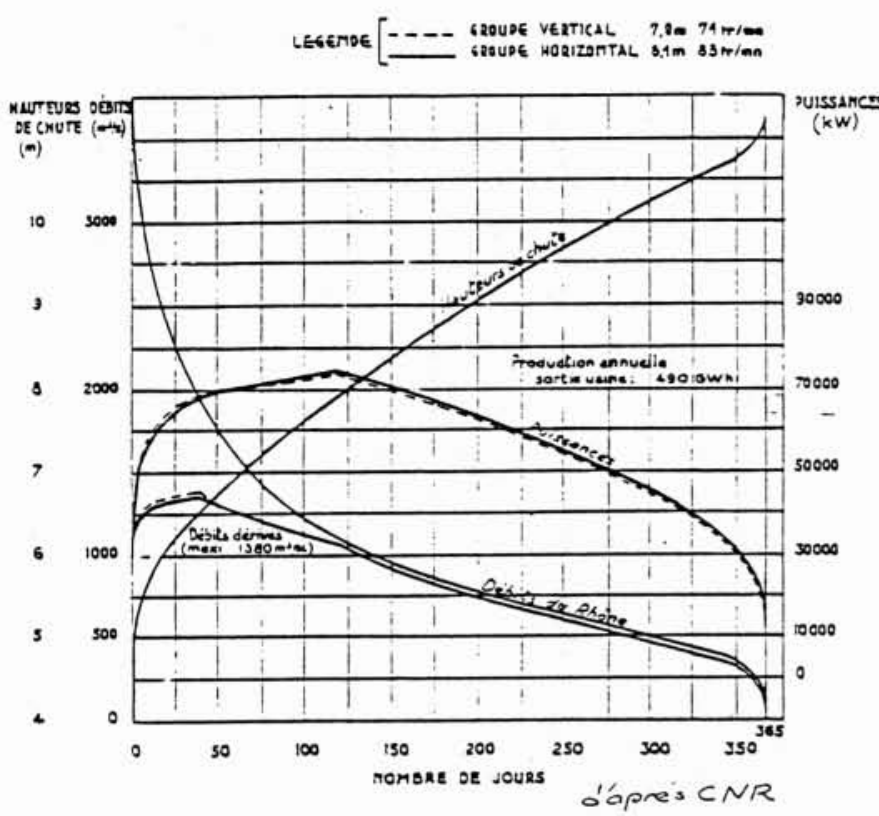

10. Caractéristiques de la chute, Courbes des débits, des hauteurs de chute et des puissances classés.

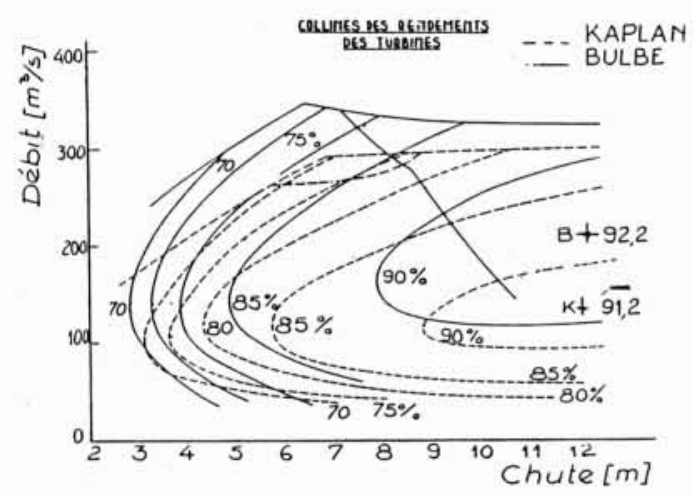

11.

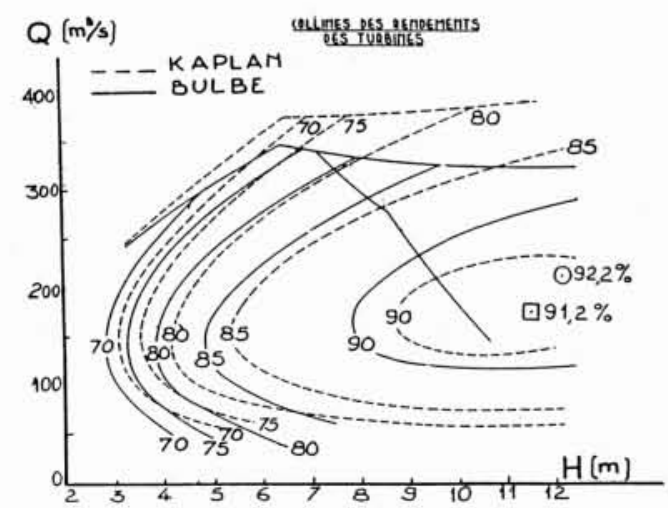

12. du groupe bulbe résiste mieux aussi à la variation de débit, surtout vers les faibles débits.

Et ceci nous amène à parler du point capital de la productivité annuelle.

Pour rester concret, nous prendrons encore l'exemple de l'usine de Pierre-Bénite étudié par P. Cazenave.

La figure 10 fait apparaître les fortes variations au cours de l'année des caractéristiques débit et hauteur de chute du fait des aléas hydrologiques du Rhône.

A partir d'essais sur modèle réduit, les collines de rendement ont pu être construites.

La figure 11 correspond à l'hypothèse de roues Kaplan et bulbe de même diamètre et tournant à la même vitesse, ce qui entraîne un net désavantage pour la roue verticale Kaplan, qui ne peut passer un débit aussi important que le groupe bulbe et fournit une puissance moindre. Un autre avantage du groupe bulbe est sa plus grande vitesse d'emballement qui lui permet de produire de l'énergie à des chutes plus faibles que les turbines Kaplan (concept de chute minimale turbinable).

La figure 12 correspond aux deux solutions Kaplan et bulbe réellement en compétition (à même débit). La turbine Kaplan tourne moins vite et est plus encombrante que le groupe bulbe. Sa colline de rendement est endessous de la colline du groupe bulbe dans tout le domaine commun de fonctionnement.

Le gain de production peut être de l'ordre de 4 millions de $\mathrm{kWh}$ sur une énergie annuelle produite de 500 millions de $\mathrm{kWh}$.

\section{Encombrement et génie civil}

Déjà bénéfique sur le plan énergétique hydraulique, le choix du groupe bulbe est aussi avantageux du point de vue de l'économie du génie civil.

Nous avons déjà vu que pour passer le même débit, une turbine Kaplan devait avoir un diamètre de roue de $13 \%$ supérieur à celui du groupe bulbe. Mais, du fait de sa conformation, la turbine Kaplan à diamètre égal est plus encombrante.

A cause de la volute de distribution, la largeur de l'amenée nécessaire pour une turbine Kaplan est, immédiatement en aval des grilles, d'environ trois fois le diamètre de la roue. Pour un groupe bulbe, cette largeur est inférieure à deux fois le diamètre de la roue.

Lorsque nous avons parlé de la cavitation, nous avons signalé que le fond de fouille devait être plus profond pour assurer à la roue Kaplan les mêmes conditions qu'au sommet de la roue du groupe bulbe.

J. Lathuile, résume la comparaison dans un tableau que nous avons complété par les indications afférentes à la turbine Kaplan équivalente en supposant que les deux machines, bulbe et Kaplan, aient le même coefficient de cavitation.

\begin{tabular}{|l|l|c|c|}
\hline & Turbine bulbe & Turbine Kaplan & $\begin{array}{c}\text { Turbine Kaplan } \\
\text { Équivalente }\end{array}$ \\
\hline Fond d'excavation & $\mathrm{H}_{\mathrm{S}}+1,5 \mathrm{D}_{\mathrm{B}}$ & $\mathrm{H}_{\mathrm{S}}+2 \mathrm{D}_{\mathrm{K}}$ & $\mathrm{H}_{\mathrm{S}}+2,25 \mathrm{D}_{\mathrm{B}}$ \\
\hline $\begin{array}{l}\text { Distance entre } \\
\text { groupe }\end{array}$ & $2,4 \mathrm{D}_{\mathrm{B}}$ & $3,4 \mathrm{D}_{\mathrm{K}}$ & $3,8 \mathrm{D}_{\mathrm{B}}$ \\
\hline
\end{tabular}


$H_{s}$ est la profondeur sous le niveau aval du point supérieur de la roue en admettant que les machines aient le même coefficient de cavitation.

Il va de soi que le point bas considéré est la cote minimale de l'aspirateur pour la turbine Kaplan et la cote minimale du conduit d'entrée au droit de l'alternateur pour le groupe bulbe (en tenant compte du fait que le diamètre de la carcasse de l'alternateur peut dépasser celui de la roue jusqu'à $30 \%$ sans altérer les performances du groupe bulbe).

Ainsi donc, quand on passe de la solution bulbe à la solution Kaplan, il y a approximativement une augmentation de $50 \%$ de l'encombrement horizontal transversal, et, à même condition de cavitation, aussi une augmentation de $40 \%$ de la profondeur maximale d'excavation. (Si on examine dans l'autre sens le passage de turbine Kaplan à groupe bulbe, il y a économie marginale de $1 / 3$ sur les encombrements horizontal transversal et vertical).

P. Cazenave chiffre à $23 \%$ l'économie sur le génie civil, directement lié aux groupes (travées de groupes et plages de montage).

\section{Souplesse et régulation}

Avantage énergétique, économie à l'investissement, mais qu'en est-il du groupe bulbe face à la turbine Kaplan dans la souplesse de fonctionnement ?

Diverses solutions peuvent intervenir dans le réglage en jouant sur l'orientation des aubes du distributeur et des pales de la roue.

Aubes et pales peuvent être fixes (à l'orientation réglée une fois pour toutes, à la meilleure conjugaison pour le fonctionnement nominal) ou mobiles (ou orientables).

Utilisons les notations :

$D$ : distributeur

$P$ : pales de la roule

$M$ : mobile

$F:$ fixe

Le double réglage $(D M, P M)$, assure évidemment la meilleure productivité annuelle, mais entraîne l'investissement le plus coûteux.

La solution sans réglage $(D F, P F)$ ou celle à distributeur fixe, exigent la présence d'une vanne aval pour la sécurité du groupe tournant.

Le tableau ci-dessous, permet une comparaison sommaire des points de vue investissement et production annuelle d'énergie.

\begin{tabular}{|c|c|c|c|}
\hline Distributeur Pales & Fixe & Mobile & \\
\hline Fixe & $\begin{array}{c}70 \mathrm{z} \\
88 \text { a } 91=\end{array}$ & $\begin{array}{c}75= \\
96 \text { × } 97=\end{array}$ & $\begin{array}{l}\text { Coût } \\
\text { Production }\end{array}$ \\
\hline Mobile & $\begin{array}{c}93 \text { z } \\
92 \text { a } 94=\end{array}$ & $\begin{array}{l}100= \\
100=\end{array}$ & $\begin{array}{l}\text { Coût } \\
\text { Production }\end{array}$ \\
\hline
\end{tabular}

En ce qui concerne les prix, il s'agit de la turbine seule. Bien entendu pour le distributeur fixe, il faut ajouter le prix de la vanne aval soit environ $10 \%$ avec les mêmes références.
La solution distributeur fixe-pale mobile serait donc celle qui conduit au coût marginal le plus faible pour le $\mathrm{kWh}$ produit. Elle présente aussi l'avantage de la sécurité au déclenchement, car à l'emballement, les pales se réorientent et peuvent laisser passer un fort débit sans un accroissement trop préjudicable de la cavitation. Au contraire, pour une machine à grande ouverture de pales fixes, le débit à l'emballement qui peut dépasser deux fois le débit nominal, provoque une forte cavitation, d'oû de fortes fluctuations de pression qui engendrent des vibrations.

Mais c'est surtout l'action de la vanne aval qui permet, aussi bien en $D F$ (où elle est obligatoire) que en $D M$ (où elle est facultative) de réduire les sollicitations sur la machine en réduisant la chute sur la roue. Pour les groupes $D M-P M$, non équipés de vanne aval, il faut surtout éviter de déconjuger pales et vannage lors de l'arrêt d'urgence. Il est donc important de prévoir des temps de manœuvres des pales tels que les deux organes ferment simultanément.

$\mathrm{Si}$ on ne considère que le fonctionnement permanent, comme un aménagement comprend généralement plusieurs groupes, on peut choisir un équipement hybride.

Par exemple, sur le Rhône, les centrales de Caderousse et de Vaugris, comportent chacune deux groupes à double réglage et deux groupes sans réglage.

Au moment de la mise en route d'un groupe bulbe sans réglage, l'ajustement de la vitesse de rotation ou synchronisation pour effectuer le couplage sur le réseau, est assurée grâce à la vanne aval commandée électroniquement.

Cette vanne aval intervient aussi pour améliorer les conditions de fonctionnement en déchargeur des groupes bulbes, soit au moment des crues, soit à la suite d'un déclenchement pour diminuer l'amplitude des intumescences.

Après déclenchement, les turbines continuent à tourner à vide, mais en même temps se ferme la vanne aval, qui peut tomber de la moitié de sa hauteur en dix secondes, augmentant ainsi la pression à l'aval de la roue, ce qui évite ou diminue la cavitation. A $75 \%$ de la vitesse nominale, le groupe peut alors décharger jusqu'à $70 \%$ du débit nominal.

\section{Problèmes mécaniques liés à la cavitation}

A plusieurs reprises, nous avons parlé de la cavitation et des fluctuations de pression qu'elle entraîne.

Nous voudrions dire, ici, un mot des recherches actuelles en France sur la question.

Comme chacun sait, la cavitation est caractérisée par le développement de bulles ou de poches de vapeur ou de gaz du fait de l'abaissement de pression rencontré par les particules de fluides au cours de leur déplacement (figure 13). Ces dépressions sont favorisées par les grandes vitesses du fluide et les décollements dans les zones à faible pression statique. Ces créations de trous de vapeur au sein de la masse liquide sont catalysées par la présence de germe de cavitation microscopique, bulles de gaz en général. 


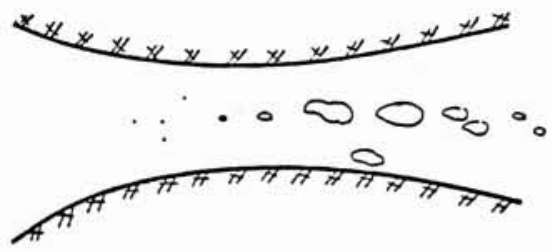

13. Génération de bulles.
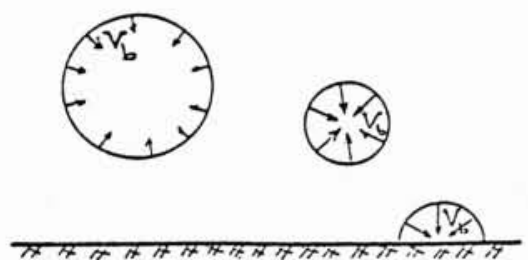

14. Implosion de bulles.

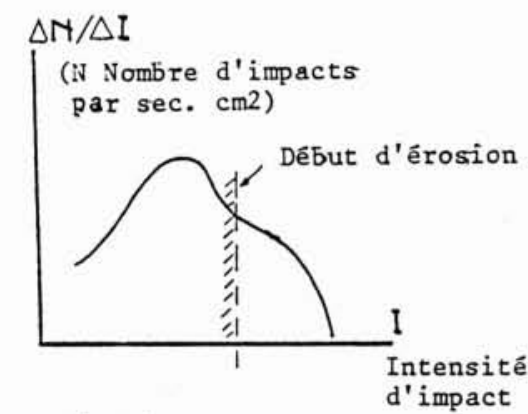

15. Fonction densité.

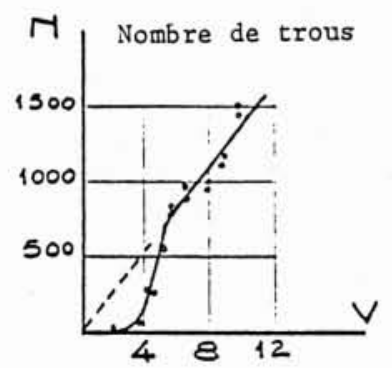

16. Résultats IMG.

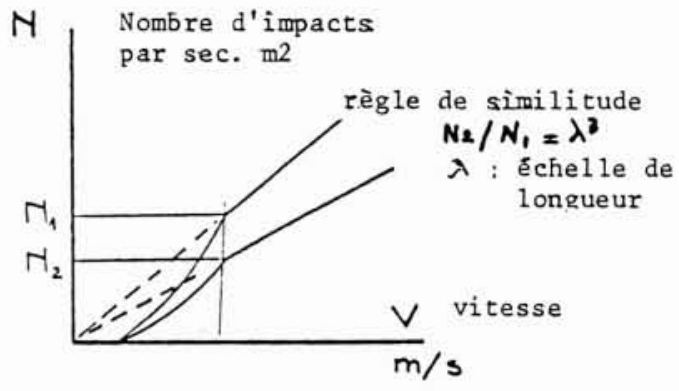

17.

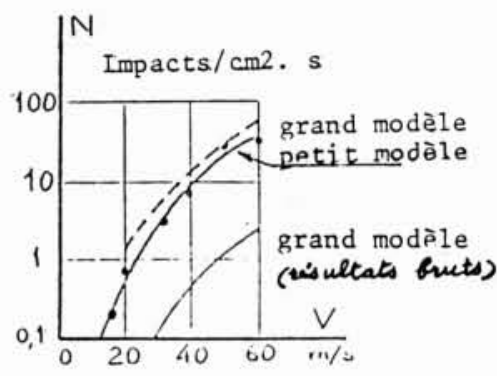

18. D'après Neyrtec,

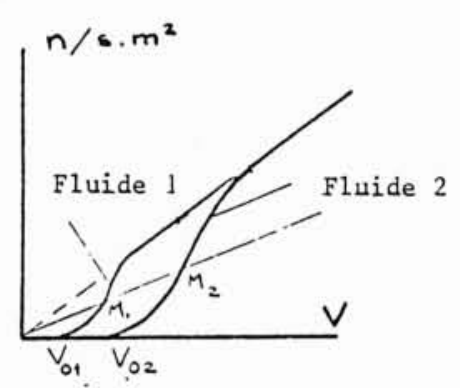

19. Influence du fluide.

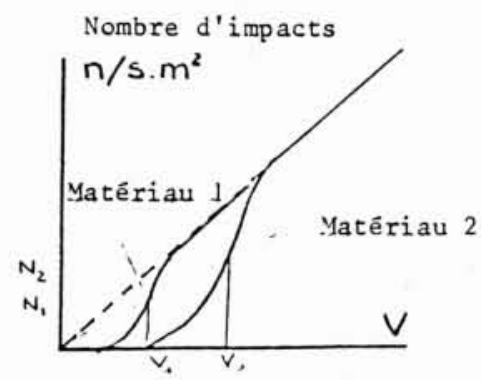

20. Influence de la nature de la paroi. Homothétie $\left(N_{2} / N_{1}\right)=$ $\left(V_{2} / V_{1}\right)$. 
Du point de vue mécanique, les ennuis ne viennent pas tellement de la production des bulles de vapeur que de leur résorption ou implosion.

En effet, la fermeture de la bulbe étant rapide, les molécules liquides près de l'interface ont une grande vitesse et, en fin de fermeture, il y a percussion d'où onde de pression à l'intérieur du liquide et choc contre l'enveloppe à la paroi (figure 14). Les chocs à la paroi se répétant nombreux provoquent l'abrasion.

Un problème important, rencontré par les fabricants de machines hydrauliques, est celui de la similitude de la cavitation et de la longueur des essais pour prévoir l'abrasion.

Une étude systématique en concertation a été entreprise en France, avec la collaboration de l'Institut de mécanique des fluides de Grenoble, Electricité de France et Alsthom Neyrtec.

Une recherche porte en particulier sur l'apparition de micro-déformations plastiques (ou trous) produites chacune par l'implosion d'une bulle de vapeur.

Ces trous sont révélateurs du phénomène d'érosion provoqué par l'écoulement cavitant. L'examen du matériau soumis aux implosions, peut donc servir à la détermination de la densité et de l'intensité des impacts ou chocs (Verry, Grison, Dorrey, 1986).

Actuellement, des essais statistiques sont menés sur la fonction de distribution des impacts sur une paroi en fonction de l'intensité caractérisée par l'ampleur du trou ou de la déformation.

L'équipe d'Electricité de France a ainsi obtenu des histogrammes tel celui de la figure schématique 15 .

Pour valider les lois de transposition de la similitude, des expériences ont été faites sur veines d'essai.

Des essais déjà réalisés, on peut tirer les conclusions suivantes :

1) Au-delà d'une certaine valeur de la vitesse du fluide, le nombre d'impacts varie linéairement comme la vitesse (figure 16),

2) Dans le cas de la cavitation à bulles, la similitude hydrodynamique exige que le rapport des teneurs en germes varie comme $\left(L_{p} / L_{s}\right)^{3}, L_{p}$ et $L_{\mathrm{s}}$ étant les longueurs homologues dans le prototype et dans le modèle $\left(L_{s} / L_{p}=\right.$ $\lambda$ (échelle géométrique)) (figure 17).

La figure 18 présente un exemple de résultats obtenus par Neyrtec sur veine fixe.

3) La teneur en germes de cavitation a peu d'influence sur la cavitation à poches.

4) Pour tenir compte de la nature du fluide, on utilise la formule classique du coup de bélier à intensité de choc égale :

$$
\rho_{1} C_{1} V_{1}=\rho_{2} C_{2} \mathbf{V}_{2}
$$

D'où la possibilité de transposition du graphe obtenu pour un fluide (du sodium par exemple) à un autre fluide (l'eau par exemple) (figure 19).

$\mathrm{Ce}$ fait a été vérifié par gammamétrie rapide sur des écoulements de sodium sur une large plage de nombre de Reynolds.

5) L'expérience a aussi montré que pour deux matériaux de paroi différents, les graphes $N, V$ se déduisaient par homothétie.

Avec cette méthode expérimentale, on peut donc prévoir très tôt une érosion future à partir de la photographie au microscope des impacts (qui pourra être traitée par l'analyse d'image).

Nous pensons aussi que la connaissance précoce des histogrammes des cratères de microdéformations doit permettre d'élaborer un modèle du type percolation pour prévoir l'érosion. L'idée de base serait de dire que la répétition des percussions en un même point provoque l'accumulation des contraintes jusqu'à l'abrasion. En discrétisant les sites, avec l'aide de l'histogramme, on peut faire l'étude stochastique de la distribution spatiotemporelle des impacts en considérant même plusieurs classes de diamètres de cratères et dire qu'il $\mathrm{y}$ a enlèvement de matière, après un certain nombre de percussions sur le même trou.

Toujours à propos de l'examen de l'abrasion, il faudrait signaler le dispositif de mesure original mis au point par le professeur Chincholle, fondé sur la mesure fine des variations d'intensité d'un courant électrique en fonction de l'épaisseur de matière érodée. Les mesures instantanées ont permis au professeur Chincholle d'étudier en veine d'essai l'érosion instantanée de cavitation en fonction du débit de la pression et de la température. Les résultats semblent mettre en évidence l'existence d'intensité maximale érosive. Des essais ont été exécutés sur une turbine de $2 \mathrm{MW}$. Ces expériences semblent intéressantes à suivre en confrontation avec d'autres procédés d'examen d'abrasion plus conventionnels.

Si l'on revient aux vibrations induites par la cavitation, il y aurait lieu d'examiner leurs conséquences sur la tenue mécanique des groupes bulbes et des précautions à prendre pour les paliers en particuliers.

Actuellement, des essais sont réalisés sur machines au Laboratoire national d'hydraulique de Chatou.

De leur côté, les ingénieurs de Neyrpic mènent simultanément des études de cavitation sur les machines en fonctionnement et sur des modèles réduits en utilisant les règles de similitude de Thoma et de Froude.

La maîtrise de ces problèmes de vibrations, fait intervenir l'analyse théorique relayée par des essais sur des machines en exploitation.

Ainsi, la Société Neyrpic bénéficie de l'expérience acquise sur plus de 150 turbines en fonctionnement, qui lui permet de progresser avec prudence et sûreté vers la réalisation d'unité de plus en plus puissantes.

Après la construction de 8 groupes bulbes de $53 \mathrm{MW}$ à Rock Island (U.S.A.), l'entreprise Neyrpic examine l'étape de groupes de $100 \mathrm{MW}$ et des diamètres de roue jusqu'à 9 mètres.

\section{Conclusions et perspectives}

Bien que cet exposé ne soit pas celui d'un spécialiste, il se proposait de montrer qu'après trente cinq ans d'expérience sur des unités de puissance importante allant jusqu'à plus de $50 \mathrm{MW}$, les groupes bulbes constituaient un concurrent efficace et économique des turbines Kaplan.

Acceptant des variations de chute impressionnantes (par exemple jusqu'à huit fois la chute minimale à la centrale de W.T. Love), s'accommodant bien de plage étendue de débit grâce au double réglage, les groupes- 
bulbes partent maintenant à la conquête des chutes légèrement supérieures à $20 \mathrm{~m}$ et du cap de $100 \mathrm{MW}$ de puissance. Suivant les informations récentes fournies par le professeur Ohashi, le projet de Tadami au nord de Tokyo prévoit la mise en service en 1990 de groupes de $64 \mathrm{MW}$.

Né en France, comme turbine-pompe, à l'usine de la Rance qui a fêté le vingtième anniversaire de sa mise en service, le groupe bulbe continuera peut être sa fonction androgyne dans des installations réversibles en région à faible relief ou en parallèle de grandes écluses en région semi-aride.

Mais, tel quel, avec ses qualités d'économie, d'encombrement modéré, d'écoulement harmonieux et de maintenance bien éprouvée, le groupe bulbe est le partenaire idéal des innombrables basses chutes, assurant une excellente décentralisation de la mise à disposition de l'énergie électrique.

\section{Remerciements}

La préparation de cette conférence a été grandement facilitée par l'accueil cordial et les informations donnés par les spécialistes d'Electricité de France, de la Compagnie nationale du Rhône, des sociétés Neyrpic, Bergeron et Guinard. La lecture critique du manuscrit et les suggestions de MM. Cazenave, Bosc et Strohl ont largement participé à l'amélioration de la rédaction.

L'auteur a aussi bénéficié des travaux et des archives de la Société hydrotechnique de France, qui a stimulé efficacement plusieurs études sur le fonctionnement a débit partiel, la cavitation et les vibrations dans les machines hydrauliques.

Enfin, il a beaucoup profité des conversations amicales avec son collègue J. Gruat, Professeur à l'Institut national polytechnique de Toulouse.

\section{Références}

(1970). - Evolution des équipements des centrales de basse chute (Rhône et Rhin). Colloque de la Commission Economique Européenne, Comité de l'Energie Electrique, Dubrovnik, 19-21 octobre.
Bosc and al. (1986). - Design and Manufacture of large hydraulic turbine machinery. Nuclear and power exhibition. Beising, February.

Boussuges P. et al., (1966). - La régulation de vitesse des groupes bulbes de fortes puissances. Communications présentée à la Société Hydrotechnique de France, Paris.

Casacci S.X., Chapus E.E., (1964). - The bulb turbine. ASME Paper 64 WA/FE 27.

CASACCı S., Bosc J. (1974), - La sécurité dans les turbomachines hydrauliques de grandes dimensions. Point de vue du constructeur. Revue Française de Mécanique, n 50-51, pp. 5-21.

Cazenave P., (1983). - Un exemple d'aménagement de basse chute équipé de groupes bulbes: Pierre-Bénite sur le Rhône. Communication présentée au Congrès de Brest, 25 septembre.

Cincholle L., Guyomard D., (1980). - Détection de la cavitation érosive et de labrasion, Symposium AIRH. Tokyo.

CinCHOLle L., (1983). - Répartition du courant à la surface d'une électrode plongée dans un milieu aqueux lorsque cette surface est partiellement abrasée. Compte-rendu Académie des Sciences - Electrochimie, t. 296 (6 juin).

Cotillon J., (1973). - Les groupes bulbes. De Röstin en Avignon : l'essor d'une technique. La Houille Blanche $\mathrm{n}^{\circ} 2 / 3$, pp. $179-200$.

Cotillon J., (1977). - Advantages of bulb unit for low heads developments. Water Power and Dam Construction, janvier.

GaUtheron J., ROCHE N., (1982). - Les turbomachines hydrauliques. Annales des Mines, $\mathrm{n}^{\circ}$ octobre, pp. 1-16.

LATHUile J. ( ). - Conception et évolution des groupes bulbes. Rapport Interne Neyrpic, 20 pages, 16 planches.

Martin G., Giraud H., (1960). - La séparation des pertes dans une turbine Kaplan de conception classique et dans une turbine axiale. Symposium Nice, 16-20 septembre.

NUESSLI W. (1969). - Flumenthal - a swiss station equipped with three $8 \mathrm{MW}$ tubular turbines.

Strohl. T., (1984). - Comparative study of bulb and Kaplan turbines for heads up to $20 \mathrm{~m}$. Internal report NEYRPIC IT/PR, 18th May.

Verry A., Grison P., Dorey J.M., (1986). - Méthodologie de prévision de l'érosion de cavitation dans les pompes. Document EDF, Direction des Etudes et Recherches, HP 416/C 01/86 01 P $47 / 2200 / 8601$. 